\title{
Mechanisms by which Organic Solvent Exchange Transforms \\ Responsive Pure Protein Hydrogels into Responsive Organogels
}

Natasha Lynn Smith, Andrew Eagle Coukouma, Ryan S. Jakubek, ${ }^{*}$ Sanford A. Asher

Department of Chemistry, University of Pittsburgh, Pittsburgh, PA 15260

*Corresponding author, email: asher@ pitt.edu

\section{Table of Contents}

1. Cryo-SEM Sample Holder

2. UVRR Spectra of BSA Hydrogels and BSA Monomers in Aqueous Solutions

3. UVRR Measurements and Protein Secondary Structure Determination

4. Water Content in BSA Organogels

a. NIR Absorbance Experimental Details

b. Calibration Curve for Water in EG

c. Calculation of Water Molecules/BSA Molecules

5. Hydrophilic BSA Polymers Retain Much of Their Hydration Shell Waters in EG

6. References 


\section{Sample Holder for Cryo-SEM Frozen Hydrated Samples}

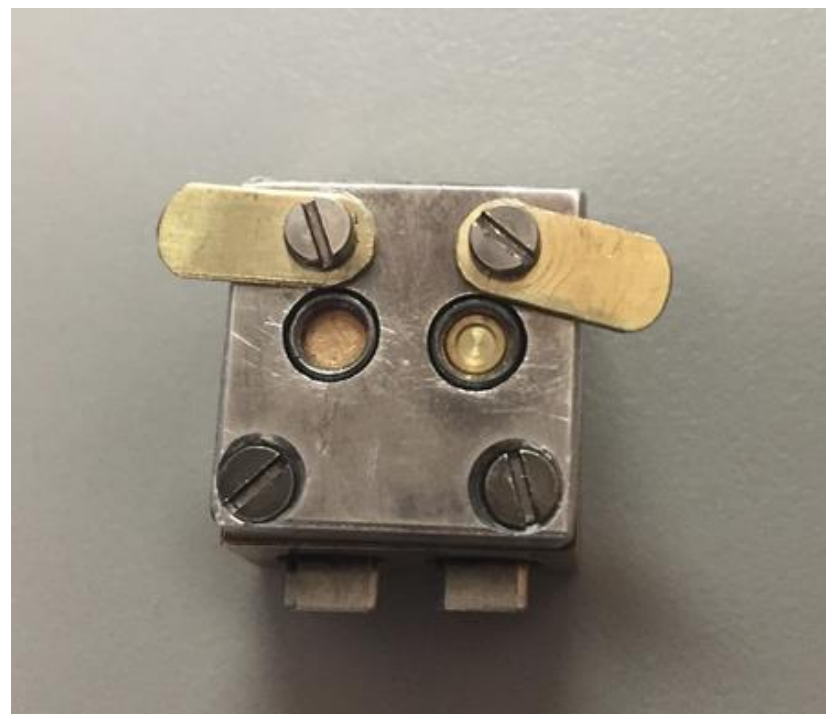

Figure SI 1. Cryo-SEM sample holder. The sample cavity on the right contains one of the Ted Pella brass planchets that is used to freeze and fracture the hydrogel or organogel samples. The sample cavity on the left is empty.

\section{UVRR Spectra of BSA Hydrogels and BSA Protein Monomers in Aqueous Solutions}

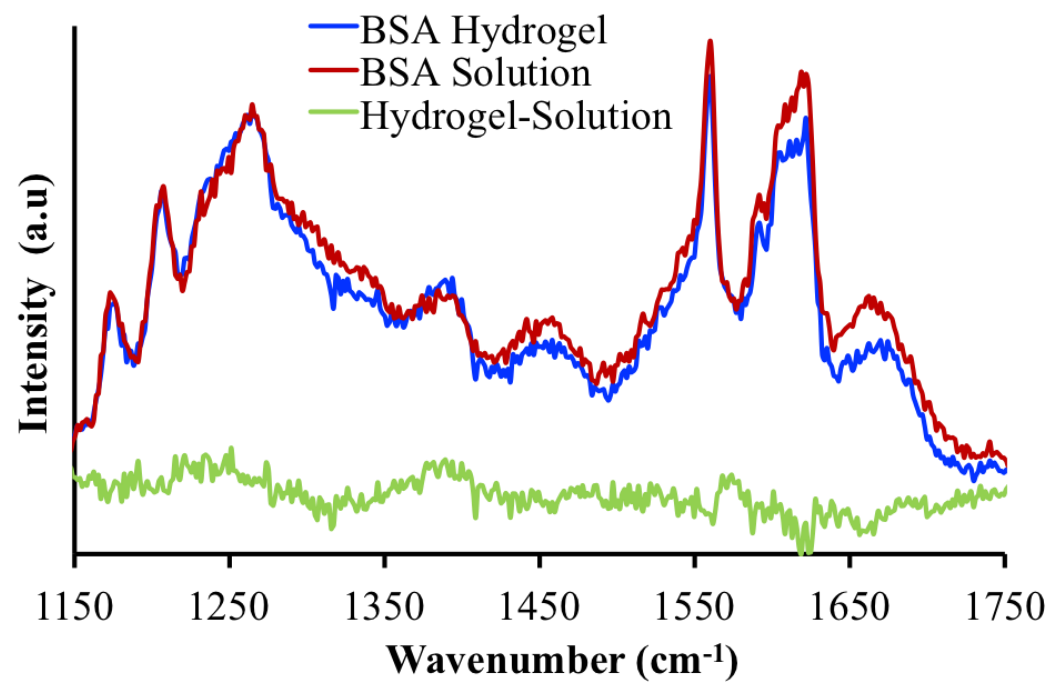

Figure SI 2. UVRR spectra of BSA hydrogel (blue), BSA protein monomer solution (red), and BSA hydrogel-monomer difference spectrum (green). 


\section{UVRR Measurements and Protein Secondary Structure Determination}

\section{Fitting the BSA UVRR Spectra}

We used Grams software (version 8.0, Thermo Fisher Scientific, Inc. Waltham, Mass., USA) to model our UVRR spectra as a sum of Gaussian and Lorentzian bands:

$$
f(x)=\sum_{i}(1-M)\left(H e^{-\left(\frac{x-x_{0}}{w}\right)^{2}(4 \ln (2))}\right)+(M)\left(\frac{H}{4\left(\frac{x-x_{0}}{w}\right)^{2}+1}\right)
$$

where $\mathrm{H}$ is the peak height, $\mathrm{X}_{0}$ is the peak frequency, $\mathrm{w}$ is the full width at half height, $\mathrm{M}=1$ if the band is Lorentzian, and $\mathrm{M}=0$ if the band is Gaussian for the $\mathrm{i}^{\mathrm{th}} \mathrm{UVRR}$ band. The $\mathrm{AmIII}_{3}{ }^{\mathrm{S}}$ fitted bands were modeled as Gaussian bands because they are inhomogenously broadened due to the peptide $\Psi$ angle distribution. ${ }^{1}$

\section{$\Psi$ Ramachandran Angle Calculation}

Details describing the methodologies for calculating $\Psi$ angles are reported by Mikhonin et al. ${ }^{1-2}$ We use the following equation to calculate the $\Psi$ angle for the $\alpha$-helix $\mathrm{AmIII}_{3}{ }^{\mathrm{S}}$ bands:

$$
v_{i}=1244 \mathrm{~cm}^{-1}-54 \mathrm{~cm}^{-1} \sin (\Psi+26)
$$

where $v_{\mathrm{i}}$ is the $\mathrm{AmIII}_{3}{ }^{\mathrm{S}}$ frequency and $\Psi$ is the backbone Ramachandran $\Psi$ angle. As discussed by Mikhonin et al., ${ }^{1}$ this equation is used to calculate the $\Psi$ angles for the peptide backbones involved in backbone-backbone hydrogen bonding. ${ }^{1}$

\section{Estimation of the Change in BSA Organogel $\alpha$-Helix Populations}

To estimate the change in protein secondary structure in the BSA organogel, we divided the total intensity of the $\mathrm{AmIII}_{3}$ bands in BSA by the intensity of the hydrogel-organogel difference spectrum. We find that the intensity of the hydrogel-organogel difference spectrum is $\sim 4 \%$ that of the total intensity of the $\mathrm{AmIII}_{3}$ band. Therefore, we estimate that the organogel contains $\sim 4 \%$ more $\alpha$-helical peptide bonds compared to that of the hydrogel. 


\section{NIR Absorption of Ethylene Glycol-Water Solutions and BSA Organogels}

Small volumes of water were added to $1.6 \mathrm{~mL}$ aliquots of EG. The EG aliquoits were directly taken from the final EG solution in which the organogels were equilibrated. During the solvent exchange, the organogels were placed on a shaker to mix the solutions. The exchange to pure EG took place over 2 days, in which $\sim 250 \mathrm{~mL}$ of pure EG was replaced 3 times daily to remove bulk water in the samples.

The 1400-2000 nm NIR absorption spectra of ethylene glycol (EG) solutions containing 0, $5,10,15,20$, and $25 \mu \mathrm{L}$ water are shown in Figure SI 3a. EG-water solutions were measured in a 5 mm path length quartz cuvette. Water has strong absorption peaks at 1400 and 1900 nm that are used to monitor the samples water content. ${ }^{3,4-5}$ These absorption bands derive from the first overtone of the $\mathrm{OH}$-stretching band $(\sim 1400 \mathrm{~nm})$ and the combination of the $\mathrm{OH}$-stretching band and the O-H bending band $(\sim 1900 \mathrm{~nm}){ }^{6}$

We utilize the $\sim 1900 \mathrm{~nm}$ peak to monitor the water content in the mobile phase because EG has stronger absorption around $1400 \mathrm{~nm}$ due to its $\mathrm{O}-\mathrm{H}$ stretching mode. EG has minimal absorption at $\sim 1900 \mathrm{~nm}^{7}$ Pure EG in a $5 \mathrm{~mm}$ pathlength cuvette shows an absorbance of 0.7 at 1915 nm. ${ }^{7}$ We measured an absorbance of 0.74 at $1915 \mathrm{~nm}$ for the EG extracted from the bottle containing the organogels, corresponding to $0.08 \mathrm{M}$ water in the bulk EG.

The difference spectrum of the EG/water solutions with the pure EG spectrum subtracted is shown in Figure SI 3b. The increasing absorbance from the increasing water concentration at $\sim 1400$ and $\sim 1900 \mathrm{~nm}$ is clearly evident. The $1915 \mathrm{~nm}$ absorbance of the EG/water solutions from this difference spectrum (Figure SI 4) was used to calculate the molar absorptivity, $\varepsilon$, of water using the Beer-Lambert equation. The $1915 \mathrm{~nm}$ molar absorptivity coefficient of water was calculated to be $\varepsilon$ $=0.95 \mathrm{M}^{-1} \mathrm{~cm}^{-1}$. 


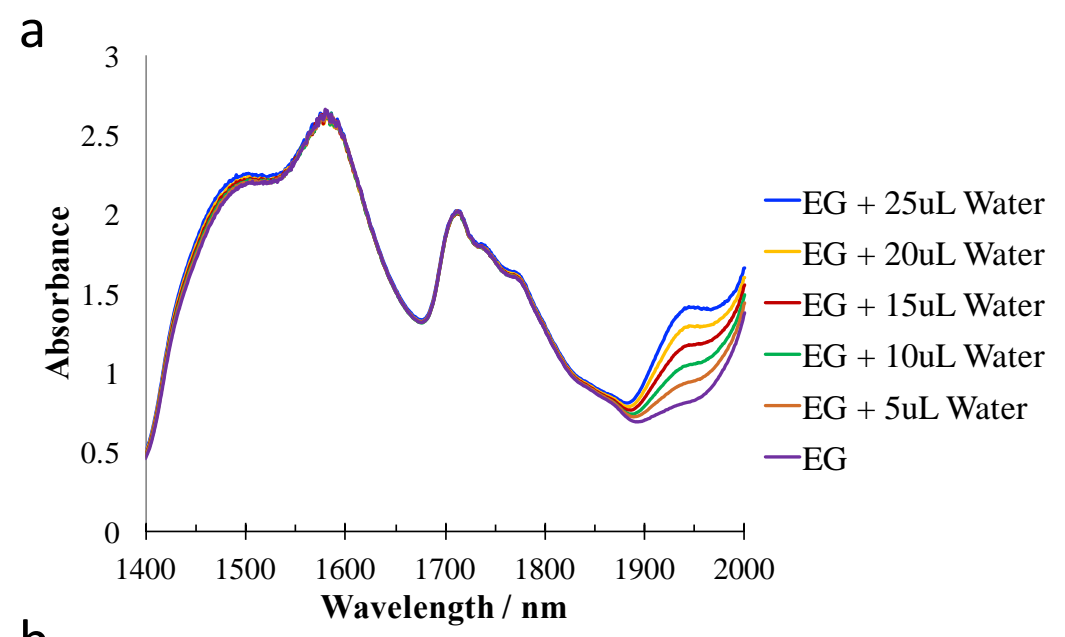

b

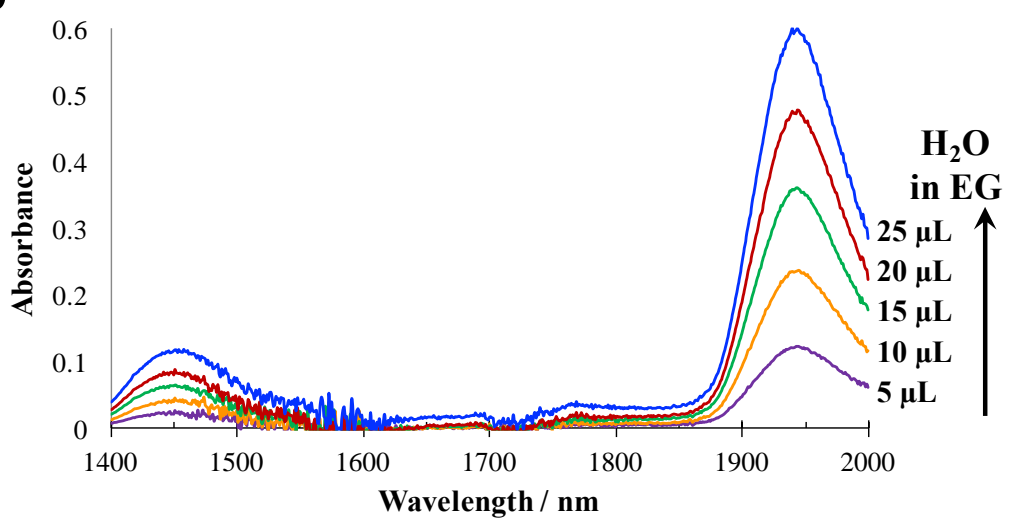

Figure SI 3. NIR absorbance of EG and its water solutions in a $5 \mathrm{~mm}$ path length quartz cuvette between $1400 \mathrm{~nm}$ to $2000 \mathrm{~nm}$. (a) Spectra of pure EG with 0, 5, 10, 15, 20, and $25 \mu \mathrm{L}$ water added

(b) Difference Spectra of (EG/water solution) - EG spectra.

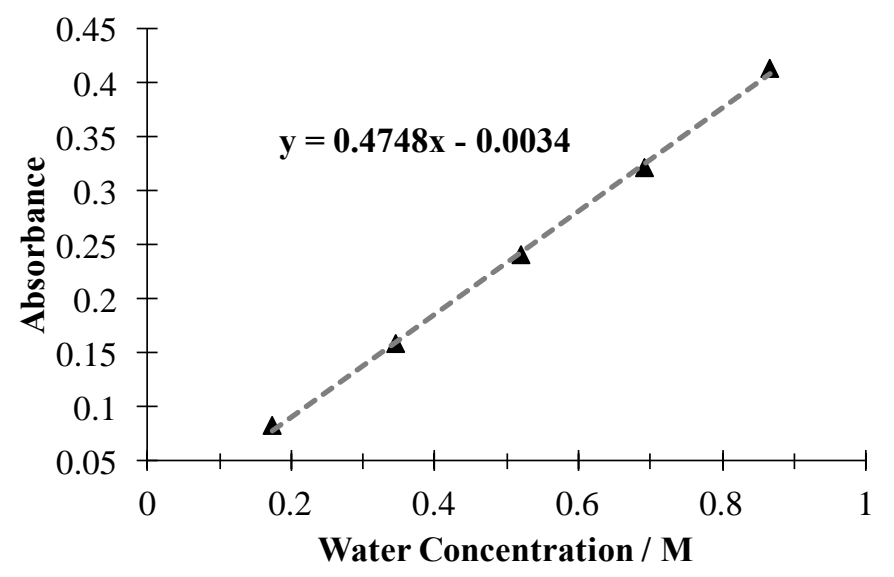

Figure SI 4. (EG+water)-EG difference spectra absorbance at $1915 \mathrm{~nm}$ as a function of water concentration. The slope of the best fit line is 0.47 A.U. $\mathrm{M}^{-1}$. 
The $1400-2000 \mathrm{~nm}$ NIR absorbance of our BSA organogels is shown in Figure SI 5. The organogel films are $420 \mu \mathrm{m}$ thick such that the double film layer is $840 \mu \mathrm{m}$ thick. The $1915 \mathrm{~nm}$ absorbance of these organogels are 0.32 for the single film layer and 0.63 for two film layers. These absorbance values yield organogel water concentrations of 8.1 $\mathrm{M}$ for a single organogel film and 7.9 $\mathrm{M}$ for two organogel films (average 8.0 $\pm 0.2 \mathrm{M}$ water).

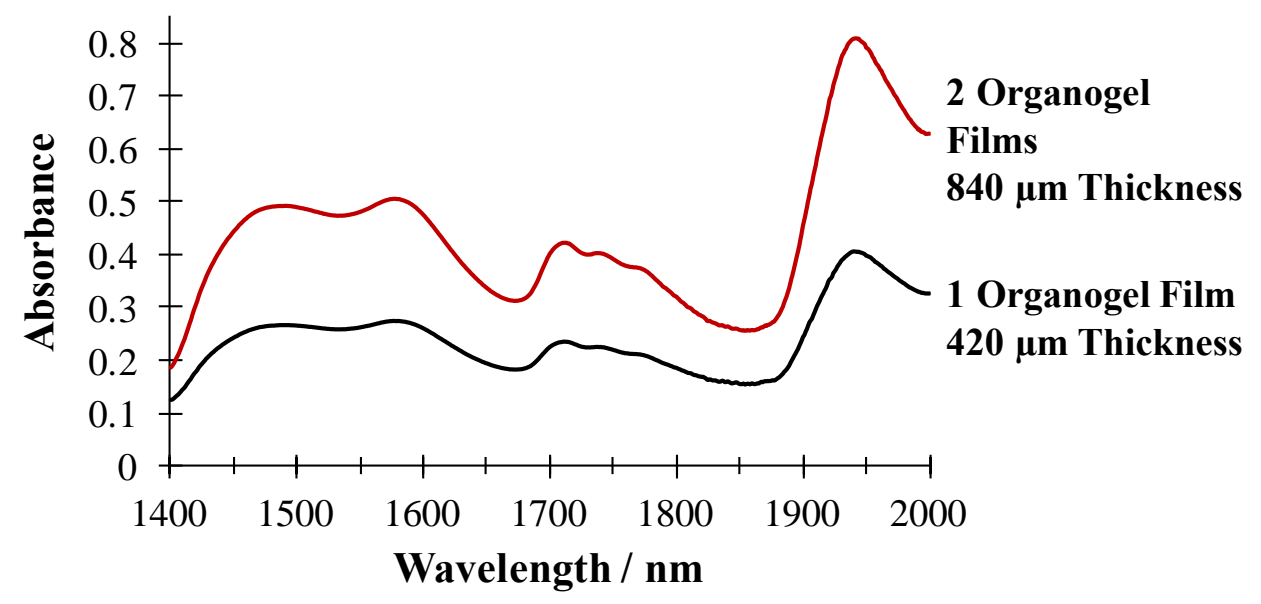

Figure SI 5. The 1400-2000 nm NIR absorbance of a single and double layer BSA organogel film.

To account for the small EG absorbance at $1915 \mathrm{~nm}$ in the organogel, we subtracted the absorbance of a pure EG film having a thickness of either $420 \mu \mathrm{m}$ or $840 \mu \mathrm{m}$. A pure EG film of $420 \mu \mathrm{m}$ thickness has a theoretical absorbance of 0.065 and an $840 \mu \mathrm{m}$ thick EG film has an absorbance of 0.13 . The $1915 \mathrm{~nm}$ absorbance of the organogel films decrease to $0.26(420 \mu \mathrm{m})$ and $0.50(840 \mu \mathrm{m})$ when the pure EG film absorbance is subtracted. The water concentration calculated from the EG subtracted organogel absorbance is now 6.5 M for a single organogel film and 6.3 M for two organogel films (average $6.4 \pm 0.2 \mathrm{M}$ water). This subtraction overestimates the absorbance from EG in the BSA organogels because the organogels contain less EG than a pure EG film. Thus, the water concentration calculated from the subtracted organogel absorbance underestimates the water content. 
Based on these measurements, we calculate the number of water molecules per BSA protein in a $1 \mathrm{~mL}$ BSA organogel sample. The organogel has a BSA polymer volume fraction of $\phi=0.27$. The organogel protein concentration is $360 \mathrm{mg} / \mathrm{mL}$ BSA (BSA MW: $66430 \mathrm{~g} / \mathrm{mol}$ ). Thus, a $1 \mathrm{~mL}$ sample of the BSA organogel contains $3.3 \times 10^{18}$ molecules of BSA. A 1 mL BSA organogel sample that contains $6.5-8 \mathrm{M}$ water has $3.9 \times 10^{21}$ to $4.8 \times 10^{21}$ molecules of water. Therefore, the ratio of water molecules per BSA protein in the organogel is calculated to be 1182-1477 water molecules per BSA protein.

\section{Hydrophilic BSA Polymers Retain Much of Their Hydration Shell Waters in EG}

Polar solvents, such as EG, more effectively strip water from a protein surface compared to non-polar solvents due to more favorable solvation enthalpies between the water and polar organic solvent. ${ }^{8-11}$ Hydrated proteins exposed to non-polar organic solvents often retain more of their

hydration layers than hydrated proteins exposed to polar organic solvents. ${ }^{9,11}$ However, while these hydrophilic polar organic solvents can strip away water molecules that are loosely bound to the protein surface, the polar organic solvents have little effect on the tightly bound water molecules around ionic and polar amino acid residues on the protein surface..$^{9-11}$

The protein hydration shell around the crosslinked BSA proteins in the pure EG organogel is mostly retained, at least in part due to the very hydrophilic nature of BSA. Gekko et al ${ }^{12}$ experimentally demonstrated that BSA is preferentially hydrated in solutions containing $60 \%$ EG in water. As shown in Figure SI 6, the BSA surface exposed to solvent is predominately hydrophilic. The hydrophilic charged and polar amino acids are shown in blue in Figure SI 6, and the non-polar hydrophobic amino acids are shown in red. The hydration shell waters that are tightly bound to the 
large surface area of hydrophilic domains in BSA likely remain intact when the aqueous mobile phase is exchange to pure EG.

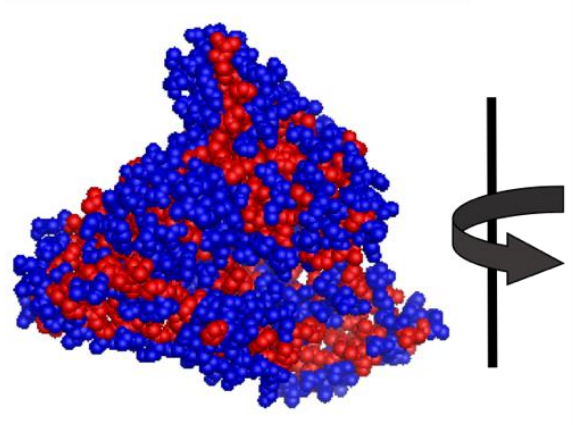

Hydrophilic

Polar and Charged

Amino Acids

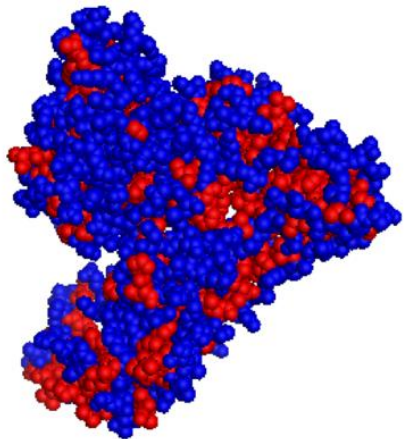

Hydrophobic

Non-Polar

Amino Acids

Figure SI 6. BSA protein structure from X-ray crystallography (PDB: 3 V03) ${ }^{13}$. Hydrophilic amino acid residues are colored blue (Lys, Arg, Asp, Glu, His, Ser, Thr, Asn, Gln, Cys); hydrophobic amino acid residues are colored red (Gly, Ala, Leu, Ile, Met, Val, Phe, Tyr, Trp, Pro).

Additionally, protein hydration shells have been protected by modifying the proteins or the protein microenvironment to enhance protein activity in organic solvents. For example, attaching highly hydrophilic groups to proteins is an effective method to protect the protein hydration shell and increase enzyme activity. ${ }^{14}$ These hydrophilic groups tightly bind numerous waters to form a protective hydrating shell around the protein, thus decreasing the ability of the organic solvent to strip waters from the protein surface. Improved protein hydration has also been demonstrated for proteins immobilized on hydrophilic materials ${ }^{15}$ and for proteins encapsulated in hydrophilic polymer networks. ${ }^{16}$ 


\section{References}

1. Mikhonin, A. V.; Bykov, S. V.; Myshakina, N. S.; Asher, S. A., Peptide Secondary Structure Folding Reaction Coordinate: Correlation between UV Raman Amide III Frequency, $\Psi$ Ramachandran Angle, and Hydrogen Bonding. J. Phys. Chem. B 2006, 110, 1928-1943.

2. Asher, S. A.; Mikhonin, A. V.; Bykov, S., UV Raman Demonstrates that $\alpha$-Helical Polyalanine Peptides Melt to Polyproline II Conformations. J. Am. Chem. Soc. 2004, 126, 8433-8440.

3. Büning-Pfaue, H., Analysis of Water in Food by Near Infrared Spectroscopy. Food Chem. 2003, $82,107-115$.

4. Tran, C. D.; De Paoli Lacerda, S. H.; Oliveira, D., Absorption of Water by Room-Temperature Ionic Liquids: Effect of Anions on Concentration and State of Water. Appl. Spectrosc. 2003, 57, $152-157$.

5. Smith, N. L.; Hong, Z.; Asher, S. A., Responsive Ionic Lquid-Polymer 2D Photonic Crystal Gas Sensors. Analyst 2014, 139, 6379-6386.

6. Luck, W. A. P.; Schiöberg, D., Spectroscopic Investigations of the Structure of Liquid Water and Aqueous Solutions. Adv. Mol. Relax. Interact. Proces. 1979, 14, 277-296.

7. GuidedWave, An Introduction to Online NIR Water Measurements in Liquid Samples. AZO Mater. [Online], Jan. 25, 2019, https://www.azom.com/article.aspx?ArticleID=17511 (accessed May, 27, 2019).

8. Sirotkin, V. A.; Sukhov, A. V.; Dudkina, E. V., Heat Effects of Dehydration of Human Serum Albumin in Hydrophilic Organic Solvents. Eng. Life Sci. 2009, 9, 74-81.

9. Yang, L.; Dordick, J. S.; Garde, S., Hydration of Enzyme in Nonaqueous Media is Consistent with Solvent Dependence of its Activity. Biophys. J. 2004, 87, 812-821.

10. Halling, P. J., High-Affinity Binding of Water by Proteins is Similar in Air and in Organic Solvents. Biochim. Biophys. Acta, Protein Struct. Mol. Enzymol. 1990, 1040, 225-228.

11. Micaêlo, N. M.; Soares, C. M., Modeling Hydration Mechanisms of Enzymes in Nonpolar and Polar Organic Solvents. FEBS J. 2007, 274, 2424-2436.

12. Gekko, K.; Morikawa, T., Preferential Hydration of Bovine Serum Albumin in Polyhydric Alcohol-Water Mixtures. J. Biochem. 1981, 90, 39-50.

13. Majorek, K. A.; Porebski, P. J.; Dayal, A.; Zimmerman, M. D.; Jablonska, K.; Stewart, A. J.; Chruszcz, M.; Minor, W., Structural and Immunologic Characterization of Bovine, Horse, and Rabbit Serum Albumins. Mol. Immunol. 2012, 52, 174-182.

14. Khmelnitsky, Y. L.; Belova, A. B.; Levashov, A. V.; Mozhaev, V. V., Relationship Between Surface Hydrophilicity of a Protein and its Stability Against Denaturation by Organic Solvents. FEBS Lett. 1991, 284, 267-269.

15. Stepankova, V.; Bidmanova, S.; Koudelakova, T.; Prokop, Z.; Chaloupkova, R.; Damborsky, J., Strategies for Stabilization of Enzymes in Organic Solvents. ACS Catal. 2013, 3, 2823-2836.

16. Zhang, P.; Sun, F.; Tsao, C.; Liu, S.; Jain, P.; Sinclair, A.; Hung, H.-C.; Bai, T.; Wu, K.; Jiang, S., Zwitterionic Gel Encapsulation Promotes Protein Stability, Enhances Pharmacokinetics, and Reduces Immunogenicity. Proc. Natl. Acad. Sci. U.S.A. 2015, 112, 12046-12051. 